\title{
Aprovechamiento de un curso de matemáticas virtual de dos generaciones en pandemia covid -19 en el 2020 y 2021
}

\author{
Judith Rocha Gámez \\ judith.rochagm@uanl.edu.mx
}

Samantha Armijo Martinez

sarmijo.me5021@uanl.edu.mx

Magdalena Escobar-Saucedo

magdalena.escobarscd@uanl.edu.mx

Facultad de Medicina UANL

Monterrey- México

\section{RESUMEN}

No cabe duda que la educación de las matemáticas ha cambiado a causa de la pandemia Covid - 19, ya que, ahora las aulas se han convertido en salas de aprendizaje virtuales. Como docentes, es un reto continuar con la enseñanza hacia nuestros alumnos, y sobre todo, asegurarnos que se lleve a cabo un aprendizaje activo. Por lo tanto, en este trabajo, se comparó el desempeño académico de dos generaciones que llevaron el curso de manera presencial, contra dos generaciones que llevaron el mismo curso de manera virtual. Para el curso virtual se hizo uso de la plataforma Microsoft Teams como aula virtual, y se realizaron actividades en simuladores y diferentes plataformas como Moodle. Se observó un mejor desempeño académico en las generaciones que llevaron el curso virtual, al presentar menor porcentaje de alumnos no aprobados en el curso $5 \%$ y $11 \%$, mientras que las generaciones que llevaron el curso presencial mostraron un $26 \%$ y $31 \%$ de alumnos reprobados. Se considera importante aumentar el uso de plataformas digitales didácticas que puedan acompañar un mejor aprendizaje en el alumno.

Palabras clave: matemáticas; desempeño; estrategias digitales. 


\title{
Taking advantage of a two generation virtual math course in covid-19 pandemic in 2020 and 2021
}

\begin{abstract}
There is no doubt that mathematics education has changed due to the Covid - 19 pandemic, since now classrooms have been turned into virtual learning rooms. As teachers, it is a challenge to continue teaching our students, and above all, to ensure that active learning takes place. Therefore, in this work, the academic performance of two generations that took the course in person was compared, against two generations that took the same course virtually. For the virtual course, the Microsoft Teams platform was used as a virtual classroom, and activities were carried out in simulators and different platforms such as Moodle. A better academic performance was observed in the generations that took the virtual course, with a lower percentage of students not approved in the course $5 \%$ and $11 \%$, while the generations that took the face-to-face course showed $26 \%$ and $31 \%$ of students failed. It is considered important to increase the use of educational digital platforms that can accompany better student learning.
\end{abstract}

Keywords: math; performance; digital strategies.

Artículo recibido: 30 noviembre. 2021 Aceptado para publicación: 29 diciembre 2021

Correspondencia: jorgeahc@live.com.mx Conflictos de Interés: Ninguna que declarar 
Aprovechamiento de un curso de matemáticas...

\section{INTRODUCCIÓN}

En el trabajo anterior "Experiencia docente en un curso de matemáticas virtual" (Rocha 2021) dimos a conocer las estrategias utilizadas para garantizar la enseñanza de las matemáticas en nivel superior en el grupo que cursa la unidad de aprendizaje de Matemáticas superior que se imparte en la licenciatura de Químico Clínico Biólogo (QCB) en la facultad de Medicina de la Universidad Autónoma de Nuevo León en forma virtual, durante el primer año de pandemia Covid-19, comparándola con grupos en forma presencial.

En el presente trabajo continuamos en pandemia y compararemos las calificaciones del último semestre agosto - diciembre 2021 con el grupo de pandemia agosto - diciembre del 2020 para observar si hay cambios o si se continúa con el mismo comportamiento de obtener mejores calificaciones llevando la asignatura de manera virtual versus presencial. Los docentes continuamos con las modificaciones hechas a nuestros programas, tareas, $\mathrm{y}$ formas de impartir la clase de manera virtual (E- Learning Masters 2017) (Márquez, G. 2020). Como profesoras de matemáticas nuestro objetivo fue aprovechar la situación para que los estudiantes desarrollaran habilidades - como leer, escribir y hablar matemáticamente - lo que nos permitió identificar áreas de oportunidad para trabajar. Blanco, M. (2012). Delgado, P. (2020)

Debemos recordar que existen diferentes formas para caracterizar un ambiente virtual. De manera general, un ambiente virtual es el modelo generado y operado en una computadora, que simula o reproduce un entorno real, en sus aspectos más importantes, de acuerdo a la finalidad de su utilización. En el campo de la educación, los ambientes virtuales facilitan la comunicación de información textual, auditiva y visual, así como su registro temporal o permanente, de manera sincrónica, o en perfecta correspondencia temporal, y asincrónica, o sin correspondencia temporal, para a apoyar el aprendizaje a distancia o en línea.

\section{Algunos autores describen las principales ventajas y desventajas de la evaluación en} ambientes virtuales, entre las que encontramos:

a) se incentiva el desarrollo de destrezas importantes en los actuales entornos económicos y sociales, como son la comunicación, el trabajo en equipo y el pensamiento crítico; 
b) se reduce tiempos y costos, al facilitar el uso de técnicas para evaluar grupos más numerosos y diversificados;

c) se posibilita el desarrollo de nuevas formas de evaluación y su integración con otras actividades del aprendizaje, así como una retroalimentación inmediata de sus resultados;

d) se ofrecen mayores oportunidades para practicar los conocimientos y destrezas adquiridas,

e) y la principal desventaja de la evaluación en un ambiente virtual o a distancia, suele ser el de la fiabilidad, dado que "puede inducir al plagio" (E- Learning Masters 2017).

La dificultad de impartición de la cátedra de matemáticas superior a dos grupos de 40 alumnos cada uno de forma virtual, se plantea abordar mediante la ejecución de ejercicios en clase y la explicación de estos y su procedimiento, haciendo uso de plataformas digitales para que la clase sea más atractiva, con participación de cada uno de los alumnos y que se pueda evidenciar el aprovechamiento de los alumnos con dicha modalidad. Se continuó utilizando la plataforma Moodle como comunicación y soporte técnico para las actividades y la plataforma MS Teams para las clases virtuales, así como la entrega de evidencias ponderables y la presentación de exámenes, para las actividades en clase se utilizó la aplicación MS Teams.

\section{ESTRATEGIAS METODOLÓGICAS O MATERIALES Y MÉTODOS}

Con dos cursos virtuales de matemáticas en el ciclo del 2020 y del 2021 se realizaron comparaciones entre las calificaciones obtenidas con el grupo vs dos grupos de años anteriores 2019 y 2018 quienes cursaron la unidad de aprendizaje en forma presencial.

Se continúa la aplicación de la misma metodología que se aplicó en el ciclo anterior en forma virtual la cual consiste en la utilización de la plataforma Microsoft Teams como aula virtual para llevar a cabo las sesiones teóricas del curso de Matemáticas superior que se imparte en el primer semestre de la licenciatura de QCB con duración de 60 minutos. En la plataforma MS Teams se realizó la formación de subgrupos, recordado que los grupos de teoría son de 40 alumnos (en cada equipo de TEAMS, estos subgrupos o canales se establecieron como "salas" 6 en este caso) en donde al final de cada sesión (15 min aproximadamente, antes de terminar) se les invitaba a abandonar la sesión general y entrar a su sala de trabajo. Así el docente entraba a cada sala a petición de los alumnos quienes tuvieran alguna duda. 
Otra estrategia utilizada fue el recurso digital Nearpood (Torres, S. 2015), en donde en esta aplicación, el docente fue creando diferentes presentaciones que incluyen diapositivas, actividades interactivas (time to climb) donde se repasaban los conceptos o ejercicios vistos en esa sesión, así mismo también se hacía el uso de simuladores como PhET interactive simulator, para diferentes temas como lo son: ecuación de la recta, despeje de ecuaciones, ecuación cuadrática ente otros. El uso del simulador propone diferentes niveles de complejidad en los procesos de aprendizaje, para que cada estudiante identifique en qué estado se encuentran sus habilidades y conocimientos, busque constructivamente un punto óptimo que lo homogenice con el grupo (Laguna, M. 2020). Por otro lado, para la explicación de los problemas y su resolución se hizo uso de la aplicación de pizarra en la sesión de MS Teams.

Las evaluaciones (Márquez, G. 2020) como los Quiz semanales y los exámenes parciales se aplicaron utilizando la plataforma Moodle (Collí, S. 2020) para resolverlos, y Microsoft Teams simultáneamente, ésta última para que mantuvieran sus cámaras encendidas durante su examen. En la Tabla 1 se muestra la evaluación de la unidad de aprendizaje de Matemáticas Superior:

\begin{tabular}{|c|c|c|c|}
\hline \multirow{7}{*}{$\begin{array}{c}\text { Etapa } \\
1\end{array}$} & Evidencia 1. Examen de resolución de problemas con operaciones & $10 \%$ & \multirow{7}{*}{$36 \%$} \\
\hline & $\begin{array}{l}\text { Evidencia 2. Examen de resolución de problemas con algoritmos } \\
\text { algebraicos donde se involucran ecuaciones. }\end{array}$ & $10 \%$ & \\
\hline & 1.1. Cuestionario sobre el contexto de la UA. & $2 \%$ & \\
\hline & $\begin{array}{l}\text { 1.2. Ensayo sobre la importancia de la UA en el programa educativo de } \\
\text { OCB. }\end{array}$ & $2 \%$ & \\
\hline & 1.3. Mapas conceptuales indicados por el profesor. & $2 \%$ & \\
\hline & $\begin{array}{l}\text { 1.4. Resolución en el aula de problemas donde se usan operaciones } \\
\text { aritméticas y algoritmos algebraicos. }\end{array}$ & $4 \%$ & \\
\hline & $\begin{array}{l}\text { 1.5. Resolución extra-aula de problemas generales y/o del } \\
\text { campo de la química donde se usan operaciones aritméticas y } \\
\text { algoritmos algebraicos. }\end{array}$ & $6 \%$ & \\
\hline \multirow{4}{*}{$\begin{array}{c}\text { Etapa } \\
2\end{array}$} & $\begin{array}{l}\text { Evidencia 3. Examen de resolución de problemas con funciones } \\
\text { trascendentes geometría analítica y trigonometría. }\end{array}$ & $10 \%$ & \multirow{4}{*}{$22 \%$} \\
\hline & 2.1. Mapas conceptuales indicados por el profesor. & $2 \%$ & \\
\hline & $\begin{array}{l}\text { 2.2. Resolución en el aula de problemas donde se usa geometría } \\
\text { analítica, trigonometría y funciones logarítmicas y exponenciales. }\end{array}$ & $4 \%$ & \\
\hline & $\begin{array}{l}\text { 2.3. Resolución extra-aula de problemas generales y/o del campo de la } \\
\text { química donde se usa geometría analítica, trigonometría y funciones } \\
\text { logarítmicas y exponenciales. }\end{array}$ & $6 \%$ & \\
\hline
\end{tabular}




\begin{tabular}{|c|l|c|c|}
\hline & $\begin{array}{l}\text { Evidencia 4. Examen de resolución de problemas con precálculo cálculo } \\
\text { diferencial e integral. }\end{array}$ & $10 \%$ & \\
\cline { 1 - 2 } & $\begin{array}{l}\text { 3.1. Mapas conceptuales indicados por el profesor. } \\
\text { Etapa } \\
\mathbf{3}\end{array}$ & $\begin{array}{l}\text { 3.2. Resolución en el aula de problemas donde se usan limites, } \\
\text { continuidad, derivadas, integrales y ecuaciones diferenciales. }\end{array}$ & $4 \%$ \\
\cline { 2 - 2 } & $\begin{array}{l}\text { 3.3. Resolución extra-aula de problemas generales y/o del campo de la } \\
\text { química donde se usan limites, continuidad, derivadas, integrales y y } \\
\text { ecuaciones diferenciales. }\end{array}$ & $6 \%$ \\
\hline
\end{tabular}

\section{RESULTADOS Y DISCUSIÓN}

Comparando las calificaciones del semestre virtual agosto - diciembre 2020 y el del 2021 vs dos semestres anteriores de manera presencial, agosto - diciembre 2019 y agosto diciembre 2018 se observó que el número de alumnos reprobados en primera oportunidad fue solamente de 5\% (4 de 80) para el semestre virtual en el 2020 y 14\% (11 de 77) para el curso virtual 2021 y para el curso presencial del 2019 fue el $31 \%$ (25 de 80) y del 2018 un $26 \%$ (20 de 80$)$.

Tabla 2 Distribución de calificaciones obtenidas de los alumnos de la generación 2020, 2021 VS LA GENERACIÓN 2018 Y 2019

\begin{tabular}{|l|c|c|c|c|}
\hline \multirow{2}{*}{ Calificaciones } & \multicolumn{2}{|c|}{ Presencial } & \multicolumn{2}{c|}{ Virtual } \\
\cline { 2 - 5 } & $\mathbf{2 0 1 8}$ & $\mathbf{2 0 1 9}$ & $\mathbf{2 0 2 0}$ & $\mathbf{2 0 2 1}$ \\
\hline Menor a 70 & 20 & 25 & 5 & 11 \\
\hline $\mathbf{7 0 - 7 5}$ & 24 & 19 & 5 & 11 \\
\hline $\mathbf{7 6 - 8 0}$ & 20 & 18 & 15 & 11 \\
\hline $\mathbf{8 1 - 8 5}$ & 8 & 8 & 20 & 22 \\
\hline $\mathbf{8 6 - 9 0}$ & 4 & 9 & 23 & 15 \\
\hline $\mathbf{9 1 - 9 5}$ & 1 & 1 & 10 & 7 \\
\hline $\mathbf{9 6}-\mathbf{9 0 0}$ & 0 & 0 & 2 & 2 \\
\hline Total de alumnos en el curso & 77 & 80 & 80 & 79 \\
\hline
\end{tabular}

Al analizar los datos obtenidos de la comparación del rendimiento de las generaciones 2018 y 2019 que fue llevada de manera presencial vs las generaciones 2020 y 2021 la cual fue virtual o en línea, se demuestra un mejor desempeño de los alumnos en base a sus calificaciones y el porcentaje de aprobados, de esta manera, se recomienda considerar que muy posiblemente se encuentren más adecuados a la tecnología y esa es la razón de su éxito, así como el uso de recursos más variados pudo tener un impacto positivo en su 
proceso de aprendizaje. En la Figura 1 se muestran imágenes de los recursos empleados durante el desarrollo de las sesiones virtuales de la unidad de aprendizaje de Matemáticas Superior.

En la Figura 1 a 3 se muestran algunos ejemplos de las actividades realizadas en diferentes recursos como en la plataforma Nearpood (Figura 1), en la Figura 2 se muestran el tipo de reactivos planteados en Time to climb, mientras en la figura 3 se muestra el empleo de los simuladores empleados.

Figura 1 Actividades Realizadas en la plataforma Nearpood.

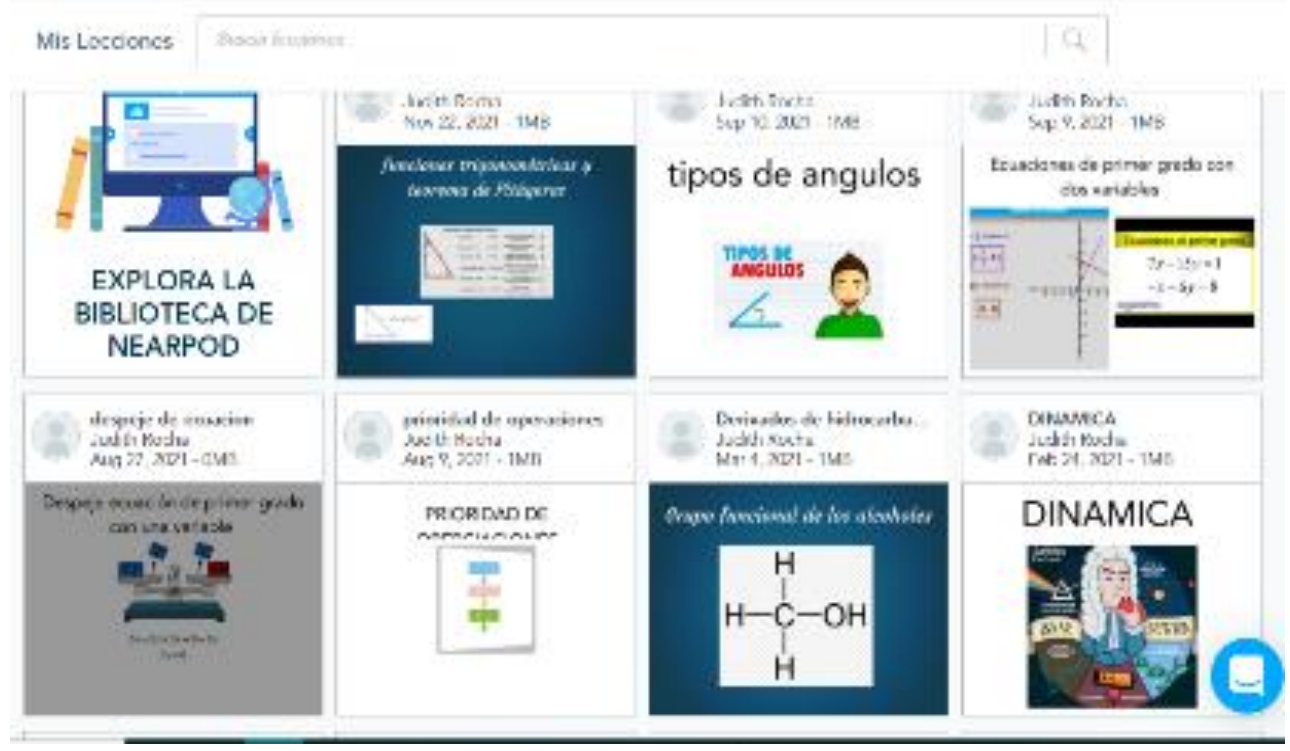

Figura 2 Uso del recurso Time to climb y el tipo de reactivos empleados

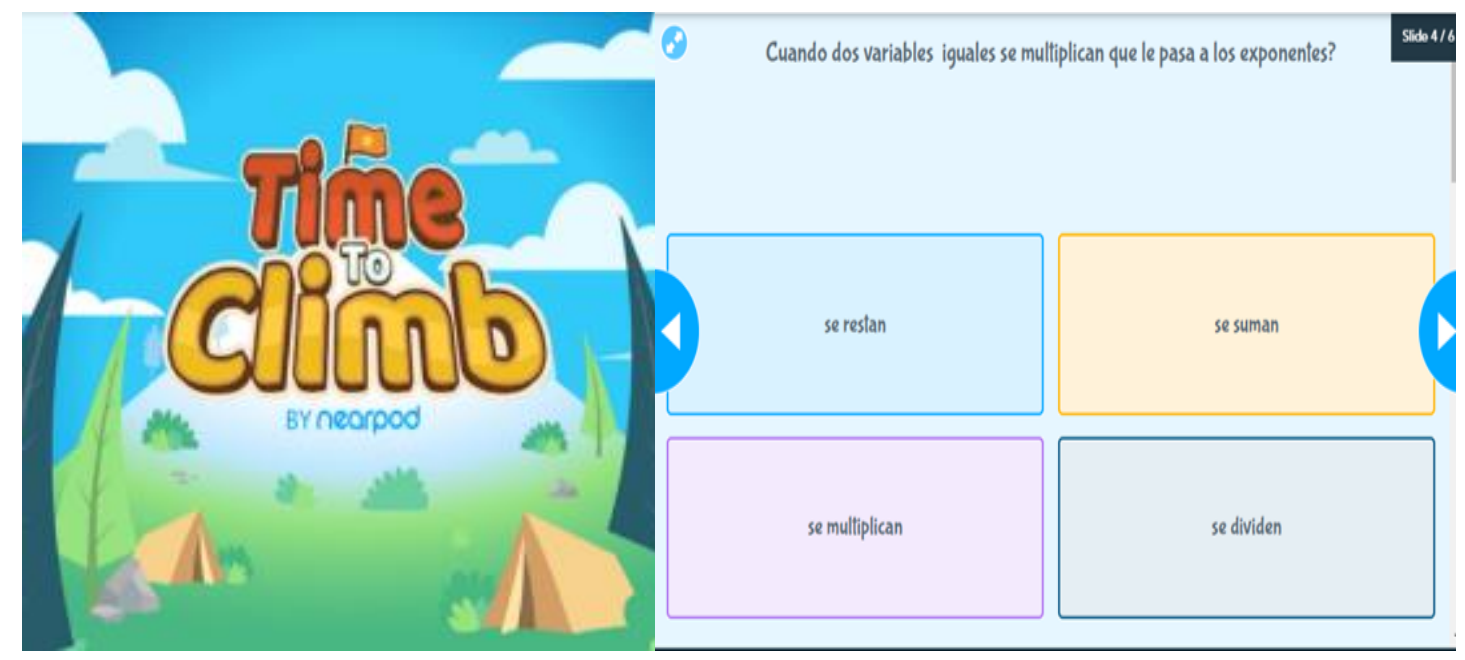


Figura 3. a) Simulador PhET utilizado, b) Niveles de complejidad propuestos, c) Simulador de ecuaciones y en d) La retroalimentación dada al estudiante.
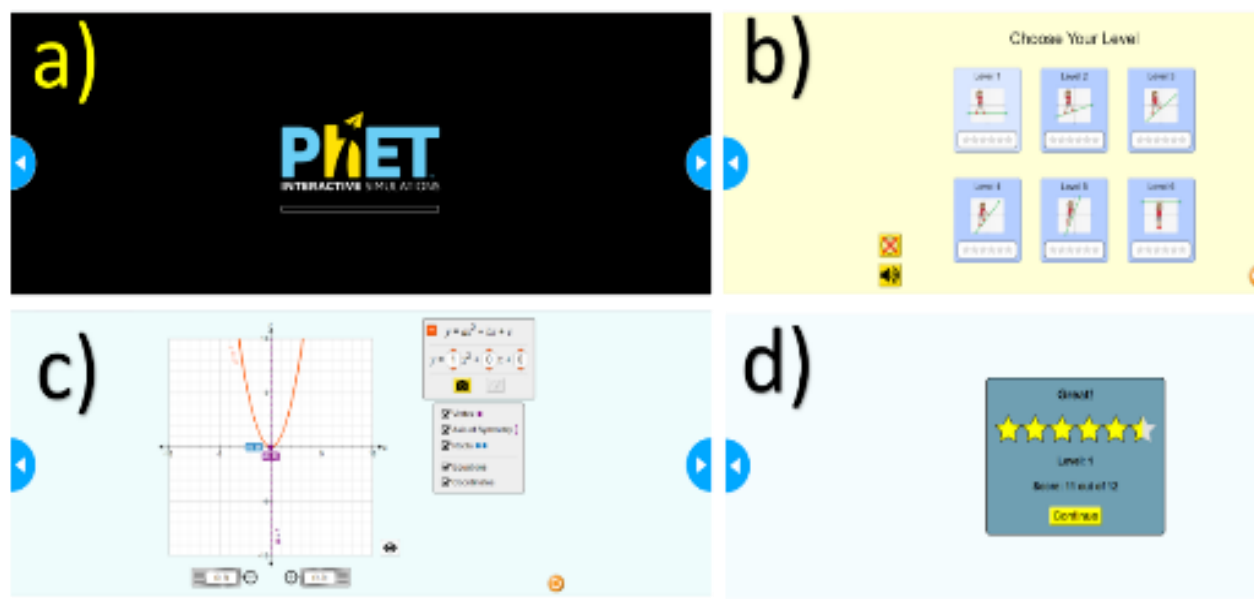

Referente al uso de los recursos adiciones se observó que el uso del simulador fue muy útil ya que el propio alumno genera las opciones de ejercicios y como le muestra una carita feliz cuando es asertiva su respuesta, y una carita triste si no es correcta, lo motiva a terminar la actividad forma personal y lo gratifica con estrellas y aplausos lo cual lo motiva en gran manera.

En cuanto a la encuesta de salida del curso de Matemáticas Superior de la Generación 2021, se muestran los resultados obtenidos en la Figura 4, mientras que en la Tabla 3 se muestran los comentarios anónimos que los alumnos hicieron respecto al curso.

1. ¿Considera que el tiempo empleado para cada tema fue el suficiente?

$\begin{array}{ll}\text { si si fue suficiente } & 55 \\ \text { en forma parcial es suficiente } & 14 \\ \text { faltó mas tiempo } & 4 \\ \text { no fué suficiente } & 1\end{array}$

2. ¿Con respecto a los ejercicios de evidencia eran claros?

$\begin{array}{lc}\text { si son muy claros } & 61 \\ \text { son claros } & 13 \\ \text { poco claros } & 0 \\ \text { nada claros } & 0\end{array}$
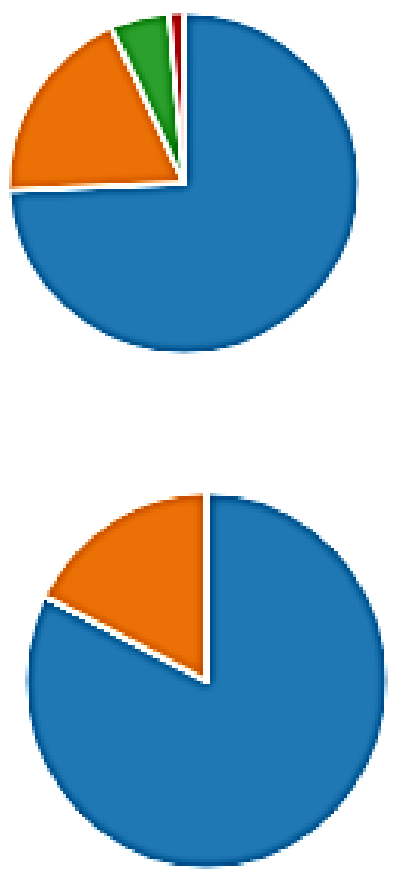
3. ¿En los quiz los reactivos están relacionados con los temas revisados?
si están relaciondos
están parcialmente relacionados 3
casi no están relacionados 0
no están relacionados
0

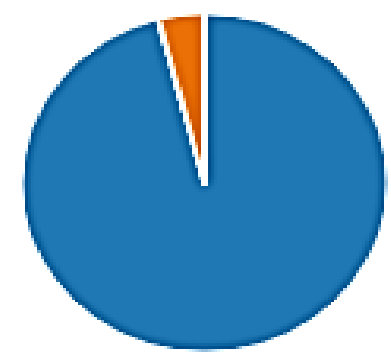

4. ¿En los exámenes parciales considera que si corresponden las preguntas a los temas revisados?

$\begin{array}{ll}\text { si corresponden totalmente } & 69 \\ \text { parcialmente corresponden } & 5 \\ \text { muy poco corresponden } & 0 \\ \text { en nada corresponde } & 0\end{array}$

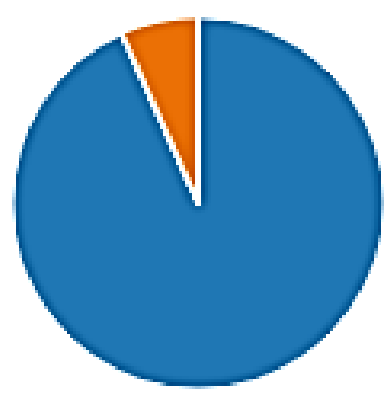

5. En los temas relacionados con ecuación de Equilibrio químico Nerst, Hasselbach, Calisus y extracción de líquidos. ¿Cómo fue su comprensión y/o calificación?
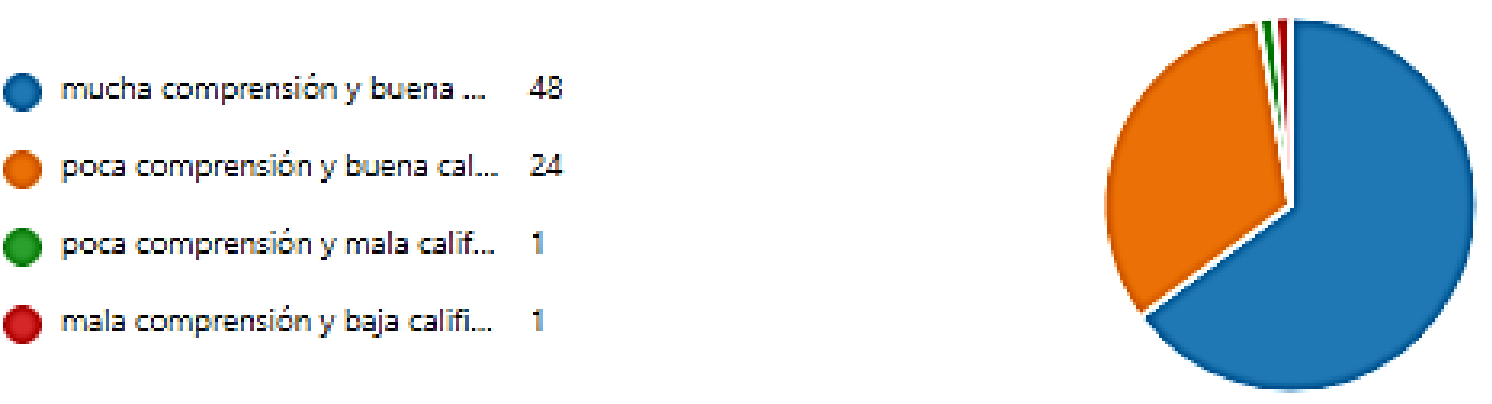

6. ¿Cómo considera la utilidad de realizar los ejercicios por equipos en sus respectivas salas en Teams?

$\begin{array}{ll}\text { muy útil } & 42 \\ \text { útil } & 21 \\ \text { poco útil } & 8 \\ \text { nada útil } & 3\end{array}$

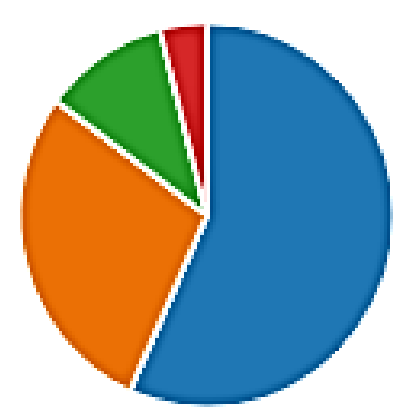


7. El realizar mapas previos a las sesiones teóricas considera usted que fue:

$\begin{array}{ll}\text { muy provechoso } & 40 \\ \text { provechoso } & 29 \\ \text { poco provechoso } & 4 \\ \text { nada provechoso } & 1\end{array}$

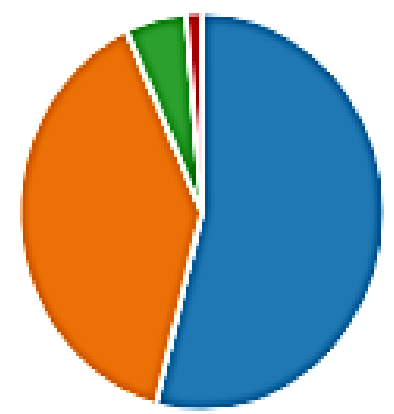

8. ¿Cómo considera la experiencia de entrevistar a los profesores de las UA de su carrera sobre la utilidad de las matemáticas?
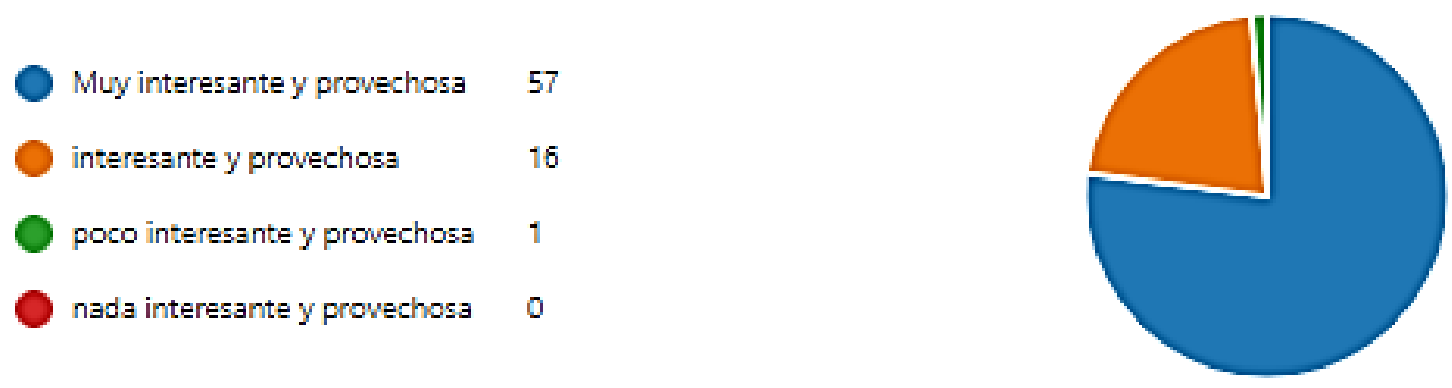

9. ¿Cómo evalúa el uso de la plataforma Nearpood en el caso de los simuladores y de la actividad Time to Climb para las actividades de los ejercicios en clase?
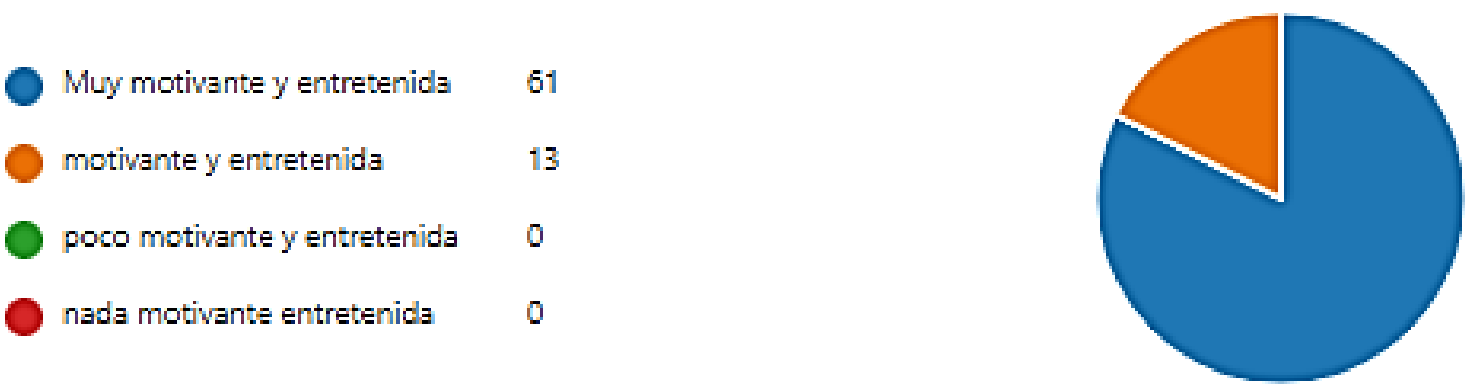

A continuación, se muestran 58 comentarios anónimos que los alumnos hicieron respecto al curso virtual de Matemáticas Superior en el año 2021:

\section{Id. Respuestas}

\begin{tabular}{|c|c|}
\hline 1 & $\begin{array}{l}\text { Me gustó mucho esta unidad, las doctoras saben manejar muy bien los temas y } \\
\text { explicarlos de manera clara }\end{array}$ \\
\hline 2 & El trabajo fue excelente, muchas gracias. \\
\hline 3 & $\begin{array}{l}\text { Tomar más tiempo para explicar temas importantes y difíciles y poner más } \\
\text { ejemplos. Ejem: derivadas, integrales, límites, etc. }\end{array}$ \\
\hline
\end{tabular}


Id. Respuestas

\begin{tabular}{|c|c|}
\hline 4 & Ninguna \\
\hline 5 & Las maestras son muy amables $(2)$ \\
\hline 6 & Me gustó mucho esta UA \\
\hline 7 & $\begin{array}{l}\text { Muy buena explicación de los temas, hacían que ejercicios difíciles se vieran } \\
\text { fáciles }\end{array}$ \\
\hline 8 & $\begin{array}{l}\text { Yo nunca había sido una persona que amara las matemáticas hasta que tuve } \\
\text { clases con las doctoras Rocha y Martínez. Excelentes maestras, siempre } \\
\text { buscando cómo solucionar los problemas que podían presentarse por el internet, } \\
\text { y siempre con mucha paciencia para nuestras dudas }\end{array}$ \\
\hline 9 & $\begin{array}{l}\text { Siento que hubo varios temas complejos que les faltó dedicarles un poco más } \\
\text { de tiempo. }\end{array}$ \\
\hline 10 & $\begin{array}{l}\text { El curso fue muy bueno, pero si considero que para algunos temas debería haber } \\
\text { más tiempo para verlos e igual realizar más ejercicios en clase participando } \\
\text { todos en resolverlos:) }\end{array}$ \\
\hline 11 & Todo excelente \\
\hline 12 & $\begin{array}{l}\text { Me gustó mucho la manera de aprender de esta materia y solo hay ciertos } \\
\text { detallitos que hay que pulir, pero todo fue muy lindo. ¡Gracias y felicidades! }\end{array}$ \\
\hline 13 & Estuvo muy padreeeeeeeeeeee, sigan así <3 \\
\hline 14 & $\begin{array}{l}\text { Muy bonita materia y muy buenas profesoras. Gracias dra Judith y profe Sam } \\
\text { por este semestre! }\end{array}$ \\
\hline 15 & $\begin{array}{l}\text { Solo pienso que al final pusieron los temas más complicados y no les dieron } \\
\text { tanta prioridad que a temas más fáciles a los que explicaron con más calma }\end{array}$ \\
\hline 16 & $\begin{array}{l}\text { Lo único que no me gustó, es que no puedes regresar a los ejercicios en el } \\
\text { examen parcial, cosa que me parece injusta ya que en forms o presenciales si se } \\
\text { puede, fuera de eso, todo increíble }{ }^{\wedge}<3\end{array}$ \\
\hline 17 & $\begin{array}{l}\text { Revisar la escritura de los ejercicios en Moodle porque a veces eran un poco } \\
\text { ambiguos al leerlos }\end{array}$ \\
\hline
\end{tabular}


Id. Respuestas

\begin{tabular}{|c|c|}
\hline 18 & $\begin{array}{l}\text { Excelente curso, las profesoras son muy buenas enseñando y siempre estaban } \\
\text { dispuestas a contestarnos nuestras dudast }\end{array}$ \\
\hline 19 & $\begin{array}{l}\text { Me gustó mucho la materia, la Dra. Judith explica muy bien y es muy } \\
\text { comprensiva, la QCB Samantha igual explicaba muy bien, muy amable y muy } \\
\text { comprensiva con nosotros los alumnos, muchas gracias por impartirnos clases:) }\end{array}$ \\
\hline 20 & Que se aborden con mayor profundidad algunos temas \\
\hline 21 & Muy buena!! \\
\hline 22 & $\begin{array}{l}\text { La verdad es que no puedo decir que la materia estuvo mala o fea, sin embargo, } \\
\text { yo casi no entiendo temas nuevos de matemáticas y pues si se me dificultó en } \\
\text { este último examen la mayoría de las preguntas. }\end{array}$ \\
\hline 23 & Muy buena materia y profesores \\
\hline 24 & Buena unidad de aprendizaje y excelentes maestras \\
\hline 25 & Me gusta que sean entregados a la materia. \\
\hline 26 & $\begin{array}{l}\text { Fue una clase muy bonita y gracias por la comprensión que nos han tenido, todo } \\
\text { el esfuerzo que hacen para darnos estas clases a pesar de las dificultades que se } \\
\text { presenten, pues sobre todo han estado muy comprometidas con nosotros y } \\
\text { hacían lo posible porque nosotros aprendiéramos, muchas gracias por todo el } \\
\text { apoyo y cariño }\end{array}$ \\
\hline 27 & Ninguna disfrute la clase de matemáticas \\
\hline 28 & $\begin{array}{l}\text { Que las maestras son increíbles solo que hay veces que los temas se ponen un } \\
\text { poco complicados y les dedicamos poco tiempo }\end{array}$ \\
\hline 29 & $\begin{array}{l}\text { Considero que los equipos deberíamos hacerlos nosotros, porque en mi caso } \\
\text { había gente no quería trabajar. Me gustó mucho la materia:))) }\end{array}$ \\
\hline 30 & $\begin{array}{l}\text { Gracias maestra la verdad ud. enseña muy bien el problema fui yo que estuve } \\
\text { algo decaída casi todo el semestre que me impidió poder concentrarme y } \\
\text { aprender más rápido por eso me iba mal en los exámenes de cada semana, } \\
\text { aunque no en todos. }\end{array}$ \\
\hline 31 & Creo que la doctora Judith es totalmente maravillosa \\
\hline
\end{tabular}


Id. Respuestas

32 Muy buena explicación de los temas, y excelentes docentes

33 Excelentes profesoras!

34 Algunos temas fueron un poco complejos y difíciles de comprender, me hubiera gustado que la enseñanza se diera en varios días y no solamente en uno.

Profe Judy y Sam, muchas gracias por sus enseñanzas, son muy buenas en su profesión y enseñan con el corazón!!! las aprecio, muchas gracias por siempre responder nuestras dudas de la mejor manera, son de esos profesores que dejan marca en los alumnos $<3$

La maestra es muy atenta, se esfuerza mucho por hacernos entender y emplea

36 todos los recursos que puede, sin embargo, la UA tiene muchos temas y siento que no se ven con el tiempo necesario para entenderlos

37 Quiero agradecerle por su buena actitud con nosotros Doctora y siempre estar cuando tenemos dudas

Todo muy bien, me gustó mucho la materia y la explicación de los temas fue muy clara

Me gustó mucho la forma en que snejola materia fue muy entretenido y pude comprender todo:) Las mejores maestras en matemáticas definitivamente

Personalmente me gustó mucho la UA, soy una persona a la que las matemáticas

40 nunca se le dieron muy bien, pero en esta ocasión si sentí que muchos de los temas se quedaron conmigo

Muy buena explicación pero faltó tiempo para revisar los temas de derivadas e integrales

Una materia muy bonita e interesante, sobre todo que las maestras hacían que

42 sintiéramos que eran temas fáciles porque lo hacían divertido y ya al final veía que dominaba un tema que si era difícil

Me gustó mucho cómo están organizados los temas, cómo se trabajan y la

43 materia en general, pero algunos temas pienso que si faltó tiempo para revisarlos o ver más ejercicios

44 Esta unidad de aprendizaje tiene a las mejores maestras en mi opinión:) 
Id. Respuestas

\begin{tabular}{|c|c|}
\hline 45 & $\begin{array}{l}\text { Me gustó mucho esta unidad, la maestra explica muy bien igual que la maestra } \\
\text { Sam }\end{array}$ \\
\hline 46 & $\begin{array}{l}\text { Considero que en su mayoría la materia está muy bien llevada a cabo, las } \\
\text { explicaciones, quiz y evidencias muy provechosas solo me gustaría que los } \\
\text { temas los practicáramos más para que quedarán mejor comprendidos los temas. }\end{array}$ \\
\hline 47 & Todo fue muy bueno y bien explicado $<3$ \\
\hline 48 & Las clases impartidas fueron muy buenas explicadas \\
\hline 49 & No usar tantos videos de YouTube para explicar \\
\hline 50 & todo muy bien \\
\hline 51 & $\begin{array}{l}\text { Todos mis comentarios para ambas maestras son positivos, ellas fueron amables } \\
\text { y también son siempre se dedicaron a nosotros, aportaron sus conocimientos y } \\
\text { en lo personal si me apoyaron en muchas cosas que antes no comprendía. Le } \\
\text { agradezco a ambas por su dedicación y sus ganas de querer aportar en nuestra } \\
\text { formación académica. }\end{array}$ \\
\hline 52 & Todo me gustó. \\
\hline 53 & $\begin{array}{l}\text { Amé mucho a mis maestras, y al usar el power como pizarrón, ayudaba mucho } \\
\text { también, muy buena materia<3 }\end{array}$ \\
\hline 54 & Excelentes clases y maestras:) \\
\hline 55 & $\begin{array}{l}\text { Todas las explicaciones fueron claras y amables, ambas maestras siempre nos } \\
\text { apoyaban }\end{array}$ \\
\hline 56 & Me gustó la materia y se me hizo divertida \\
\hline 57 & Muchas gracias por todo maestras! \\
\hline 58 & Excelente maestra \\
\hline
\end{tabular}

Con base a la encuesta se observa en los rubros donde se revisan temas relacionados con su carrera y la aplicación de ecuaciones de primer grado y despejes que presentan cierto grado de dificultad hacer esa correlación, es un área de oportunidad para remediar 
También en la encuesta manifiestan que el uso de las salitas virtuales es bueno, pero no excelente, seguramente porque el tiempo en el que el docente visita las salas es muy reducido, se tendrá que considerar dar más tiempo a esta actividad o contar con más de dos docentes durante la sesión.

\section{CONCLUSIÓN O CONSIDERACIONES FINALES}

Como se pudo constatar, continúa el comportamiento de mejor desempeño académico de alumnos de Matemáticas Superior de la carrera de Químico Clínico Biólogo al llevar a cabo la clase de manera virtual, mostrando mejores resultados y aprendizaje cuando se llevó a cabo de manera virtual mediante el uso de plataformas digitales. Se propone de manera importante aumentar el uso de plataformas digitales didácticas que acompañen el mejor aprendizaje del alumno.

No hay que olvidar que el profesor es un elemento esencial para mejorar la calidad de los procesos educativos en la metodología a distancia y virtual (Faisal 2021), en la medida que interactúe con sus estudiantes de manera empática, proactiva, motivante y dialógica a fin de favorecer la asimilación del nuevo conocimiento. Su intervención ha de estimular y orientar al estudiante, por medio de situaciones didácticas de aprendizaje y de diversas estrategias que lo ayudan a superar las dificultades encontradas al abordar una situación problema. (Juan, A. 2012) (Ortíz, E. 2020)

\section{REFERENCIAS BIBLIOGRAFICAS}

Albano, G. (2012). Conocimientos destrezas y competencias un modelo para aprender matemáticas en un entorno virtual. Revista de Universidad y sociedad del conocimiento. Universities and Knowlegde Society Journal.

Alejandre, J. L. (2013). Experiencia docente en un curso cero semipresencial. Departamento De Matemática Aplicada. Universidad de Zaragoza.

Blanco, M. (2012). Los cuestionarios del entorno Moodle: su contribución a la evaluación virtual formativa de los alumnos de matemáticas de primer año de las titulaciones de Ingeniería. Universities and Knowlegde Society Journal.

Burner J.S. Toward,(1974) a theory of instruction Cambrige, Mass: Belknap Press of Harvard,University Press.1974.

Clates (1976).Enseñanza modular E. de O. de la U.A.B.C. (1976) (pp 16) 
Collí, S. (2020). La enseñanza de las matemáticas: una reflexión sobre su transformación necesaria en tiempos de contingencia. Universidad Autónoma de Yucatán Mérida, Yucatán.

Covey Stephen, (2015) Los siete hábitos de la gente altamente efectiva, Ed. Paidos, 2015.

Craing Mehrens, (1989). Psicología educativa contemporánea, concepto, temática y aplicaciones, Ed. LIMUSA, 1989

Cuypers, H. (2012). Teaching Mathematics Online: Emergent Technologies and Methodologies, editado por Ángel A. Juan, María A. Huertas, Sven Trenholm y Cristina Steegmann» [reseña en línea]. Revista de Universidad y Sociedad del Conocimiento (RUSC). vol. 9, n. ${ }^{\circ} 1$, págs. 184-189.

Delgado, P. (2020). La enseñanza de las matemáticas requiere una urgente reestructuración, señala nuevo reporte. Observatorio Instituto para El Futuro de la Educación. ITESM.

E- Learning Masters (2017). Técnicas y estrategias de enseñanza virtual. http://elearningmasters.galileo.edu/2017/10/17/tecnicas-y- estrategias-deensenanza-virtual/

Faisal, A. Virtual Mathematics Education during COVID-19 An Exploratory study of teaching Practices for Teachers in Simultaneus Virtual Classes International Journal of Learning and Educational Research Vol 20 No. 12 pp 85-113, December 2021

Marzano, Robert.(2016) Dimensiones del aprendizaje: manual para el maestro (2a. ed.). Guadalajara, MX: ITESO - Instituto Tecnológico y de Estudios Superiores de Occidente, 2014. ProQuest ebrary. Web. 11 December 2016. Copyright (C) 2014. ITESO - Instituto Tecnológico y de Estudios Superiores de Occidente. All rights reserved.

Mendoza J.,(1981) El proyecto ideológico modernizador de las políticas universitarias en México, (1965-1980) Revista Perfiles Educativos,. CISE-UNAM, 1981

Ortíz, E. (2020). Impulsando la educación matemática a través de la tecnología durante y después de la pandemia. Blog BID, mejorando vidas.

Padilla A.(2012) El Sistema modular de enseñana: Una alternative curricular de la educación superior universitaria en México Rev. de docencia Universitara de educación superior universiaria Vol 10 Oct-Dic 2012, 71-98 
Rocha J.: (1996) La Importancia de la secuenciación de las materias del área de Química del Dpto.de Química Analítica de la Fac. de Medicina de la U.A.N.L. Julio 1996 (Filosofía y Letras U.A.N.L.)

Rocha J. (1992) Análisis Retrospectivo de las Calificaciones obtenidas en el 5to semestre de la carrera de Químico Clínico Biólogo Sept. 86 Marzo 92 Oct.1992

Rocha J (2014)“ Evaluación del error durante la preparación de soluciones: una herramienta para el apoyo de aprendizaje “ 49 Congreso Mexicano de Química 33 congreso Nacional de educación Química Mérida 2014

Rocha J (2021) Experiencia docente en un curso de matemáticas virtual en pandemia covid - 19 Ciencia Latina Revista Científica Multidisciplinar, Ciudad de México, México. octubre, 2021, Volumen 5, Número 5. p.8149 